\section{Françoise Degos Jean-Pierre Benhamou}

\section{ADRESSES}

F. Degos: praticien hospitalier. J.-P. Benhamou: chef du service hépatologie. Service d'hépatologie et Inserm U. 24, 100, boulevard du Général-Leclerc, 92110 Clichy, France.

\title{
Le traitement des hépatites chroniques
}

L'interféron $\alpha$, seul ou associé aux glucocorticoïdes, est actif dans environ $40 \%$ des cas d'hépatite $B$; il entraîne alors une inhibition de la réplication virale, une amélioration des signes histologiques et une séroconversion (passage de la positivité pour l'antigène $\mathrm{HBe}$ à celle pour l'anticorps anti-HBe). De faibles doses du même interféron semblent actives chez 80 \% des malades atteints d'hépatite $\mathrm{C}$, mais les rechutes seront fréquentes à l'arrêt du traitement, nécessitant probablement une administration prolongée dont l'efficacité et les effets secondaires doivent faire l'objet d'études cliniques et thérapeutiques complémentaires.

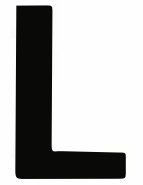

a thérapeutique des hépatites chroniques virales a fait de sérieux progrès pendant ces dix dernières années. Désormais, l'hépatologue peut abandonner l'attitude contemplative qui était la sienne devant les malades atteints d'hépatite chronique virale. Toutefois, les traitements actuellement disponibles sont encore imparfaits. D'une part, leur efficacité est inconstante : seule une fraction des malades semble en bénéficier; d'autre part, leur efficacité est souvent incomplète.

Les hépatites virales chroniques ont en commun un certain nombre de caractéristiques importantes. En premier lieu, quelle que soit la nature $\mathrm{du}$ virus responsable, les lésions histologiques sont les mêmes; ces lésions, lorsqu'elles sont sévères, entraînent une insuffisance hépatocellulaire. En deuxième lieu, les hépatite's virales chroniques font toutes courir, à plus ou moins long terme, un risque de cirrhose. En troisième lieu, surtout lorsqu'une cirrhose est constituée, il existe un risque de développement d'un car- cinome hépatocellulaire.

Sur le plan virologique, il existe trois types d'hépatite virale chronique: l'hépatite virale chronique due à l'infection par le virus $B$, l'hépatite virale chronique due à la double infection par les virus $B$ et $D$, et l'hépatite virale chronique due à l'infection par le virus $\mathrm{C}$.

\section{Hépatite virale chronique $B$}

Pour bien comprendre le mode d'action des traitements antiviraux, il convient de rappeler des notions importantes concernant le mécanisme des lésions hépatocytaires en cas d'infection virale $B$ et l'histoire naturelle de cette infection virale.

Le virus B n'entraîne pas directement des lésions de l'hépatocyte dans lequel il prolifère. Les lésions hépatocytaires sont la conséquence de la réponse immunitaire du sujet infecté. En effet, l'hépatocyte dans lequel prolifère le virus $\mathrm{B}$ exprime à sa surface des antigènes viraux, en particulier l'antigène $\mathrm{HBc}$. De ce fait, l'hépatocyte infecté, exprimant des antigènes étrangers, est la cible de 


\section{RÉFÉRENCES}

1. Chu CM, Karayannis P, Fowler MJF, et al. Natural history of chronic hepatitis B virus infection in Taiwan : studies of hepatitis B virus DNA in serum. Hepatology $1985 ; 5$ : 431.4.

2. Degos F, Marcellin P, Benhamou JP. Traitement de l'hépatite chronique active due à l'infection par le virus de l'hépatite B. Gastroenterol Clin Biol 1988; 12 : 845-54.

3. Fiume L, Torrani Cerenzia MR, Bonino F, et al. Inhibition of hepatitis B virus replication by vidarabine monophosphate conjugated with lactosaminated serum albumin. Lancet, 1988 ; 2 : 13-5.

4. Perillo RP. Antiviral therapy of chronic viral hepatitis. Curr Opinion Gastroenterol $1988 ; 4: 420-7$.

5. Davis GL, Hoofnagle JH. Interferon in viral hepatitis. Hepatology $1986 ; 6$ : 1038-41.

6. Perillo RP. Interferon therapy for chronic type B hepatitis: the promise comes of age. Gastroenterology 1989; 96 : 532-6.

7. Alexander GJM, Braham J, Fagan EA, et al. Loss of HBsAg with interferon therapy of chronic hepatitis B virus infection. Lancet $1987 ; 2: 66-9$

8. Dusheiko G, Dibisceglie A, Boywers S, et al. Recombinant leukocyte interferon treatment of chronic hepatitis B. Hepatology $1985 ; 5$ : 556-60.

9. Hoofnagle JH, Peters M, Mullen KD, et al. Randomized controlled trial of recombinant alpha interferon in patients with chronic type B hepatitis. Gastroenterology 1988 ; 95 : 1318 25.

10. Kakumu H, Fuji A, Yooshioka K, et al. Pilot study of recombinant interleukin-2 for chronic type B hepatitis. Hepatology 1988 ; 8 : la réponse immunitaire. Lorsque la réponse immunitaire à l'égard du virus $B$ est nulle, le virus prolifère et les lésions hépatiques sont minimes ; à l'opposé, lorsque la réponse immunitaire est vigoureuse, les lésions hépatocytaires sont sévères et la multiplication virale est ainsi freinée.

L'infection chronique par le virus B comporte trois phases successives [1] (figure 1). Dans une première phase, il existe une multiplication importante du virus B. Sur le plan virologique, cette phase est caractérisée par la présence de marqueurs de la multiplication virale $B$ : antigène $H B e$, ADN polymérase, ADN VHB. Sur le plan lésionnel, cette phase est caractérisée par une forte activité, c'est-àdire des phénomènes de nécrose hépatocytaire marqués par une élévation des transaminases et des phénomènes inflammatoires. Cette première phase dure quelques mois à quelques années.

Dans une deuxième phase, dite de séroconversion, la multiplication virale diminue, probablement parce qu'une réponse immunitaire finit par se produire. Sur le plan virologique cette phase est caractérisée par la diminution, puis la disparition, des marqueurs de la multiplication virale $B$ : antigène $\mathrm{HBe}, \mathrm{ADN}$ polymérase, ADN VHB; l'activité est encore plus forte que précédemment. Cette phase de séroconversion dure quelques semaines à quelques mois. Pendant la première phase de multiplication virale et la deuxième phase de séroconversion, des lésions graves, partiellement irréversibles, allant jusqu'à la constitution d'une cirrhose, se développent. En étant très schématique, on peut dire que le pronostic de l'infection virale $B$ se joue principalement dans ces deux premières phases.

Dans une troisième phase, la multiplication virale devient faible ou nulle. Sur le plan virologique, cette période est caractérisée par la disparition des marqueurs de la multiplication virale et l'apparition de l'anticorps anti-HBe. Sur le plan lésionnel, on assiste alors à la disparition des lésions actives, c'est-à-dire de la nécrose hépatocytaire et de l'infiltration inflammatoire. Pendant cette phase, principalement lorsqu'une cirrhose s'est déjà constituée, un carcinome hépatocellulaire peut se développer. Une réactivation de l'infection virale $B$, c'est-à-dire la réapparition de la multiplication virale $\mathrm{B}$, peut également se produire avec, pour conséquence, une résurgence de lésions actives.

Les traitements actuellement disponibles ne permettent généralement pas l'éradication de l'infection virale $\mathrm{B}$ (c'est-à-dire la disparition du VHB, qui se traduirait par la disparition de l'antigène $\mathrm{HBs})$. Ils permettent seulement d'obtenir une séroconversion plus précoce que celle qui se produirait en cas d'évolution spontanée. Il est raisonnable de penser que cette séroconversion précocement obtenue réduit les lésions entraînées par la multiplication prolongée du virus $B$

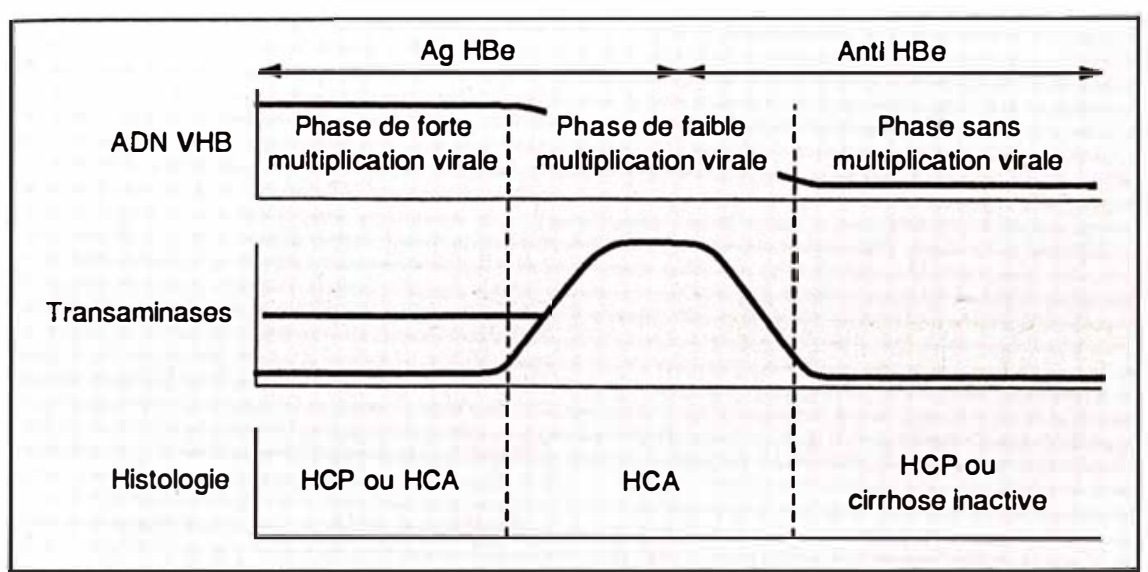

Figure 1. Histoire naturelle de l'infection chronique par le virus B. HCP $=$ hépatite chronique persistante. $H C A=$ hépatite chronique active. Au cours de la phase de forte multiplication virale, les transaminases sont généralement augmentées mais peuvent être proches de la normale. 
et représente ainsi un bénéfice pour les malades. Toutefois, un tel bénéfice, qui ne pourrait être évalué que par des études portant sur plusieurs années, n'a pas encore été démontré.

\section{Les antiviraux et les interférons dans I'hépatite B}

- La vidarabine. La vidarabine (adénine arabinoside ou Ara-A) et son métabolite phosphorylé inhibent l'ADN polymérase nécessaire à la multiplication du VHB. Cette molécule de courte durée de vie est peu soluble et doit donc être administrée sous forme intraveineuse. Le dérivé monophosphate (adénine arabinoside 5'-monophosphate ou AraAMP) est plus soluble, a la même activité antivirale et peut être administré par voie intramusculaire. Les résultats de l'ensemble des études contrôlées du traitement des hépatites chroniques liées au VHB par la vidarabine sont discordants [2].

Ces études, dans les meilleurs des cas, démontrent une inhibition durable de la multiplication virale et une séroconversion $\mathrm{HBe} / \mathrm{anti}-\mathrm{HBe}$ dans $30 \%$ des cas. Les effets secondaires de ce traitement, en particulier les complications neuromusculaires, sont fréquents et il est indispensable d'interrompre l'administration de ce médicament dès leur apparition. Le dérivé conjugué à la sérum-albumine lactosaminée, fixé exclusivement au niveau du foie, donc a priori sans effets secondaires, aurait un effet antiviral similaire à celui de la vidarabine [3].

La fréquence des effets secondaires est probablement responsable du désintérêt de nombreux auteurs pour cette molécule, l'interféron ayant en fait une activité antivirale au moins comparable et des effets secondaires plus acceptables.

- Autres médicaments antiviraux. L'aciclovir (et sa prodrogue, la 6déoxyacyclovir), la suramine et la 2', 3'-didéoxycytidine (un inhibiteur de l'activité reverse transcriptase du VIH) ont tous des effets antiviraux expérimentaux contre le VHB, mais cette efficacité n'a pas été démontrée in vivo pour l'aciclovir, et reste à démontrer pour les autres produits [4].

$\mathrm{m} / \mathrm{s} n^{\circ} 2$ vol. 6, féurier 90

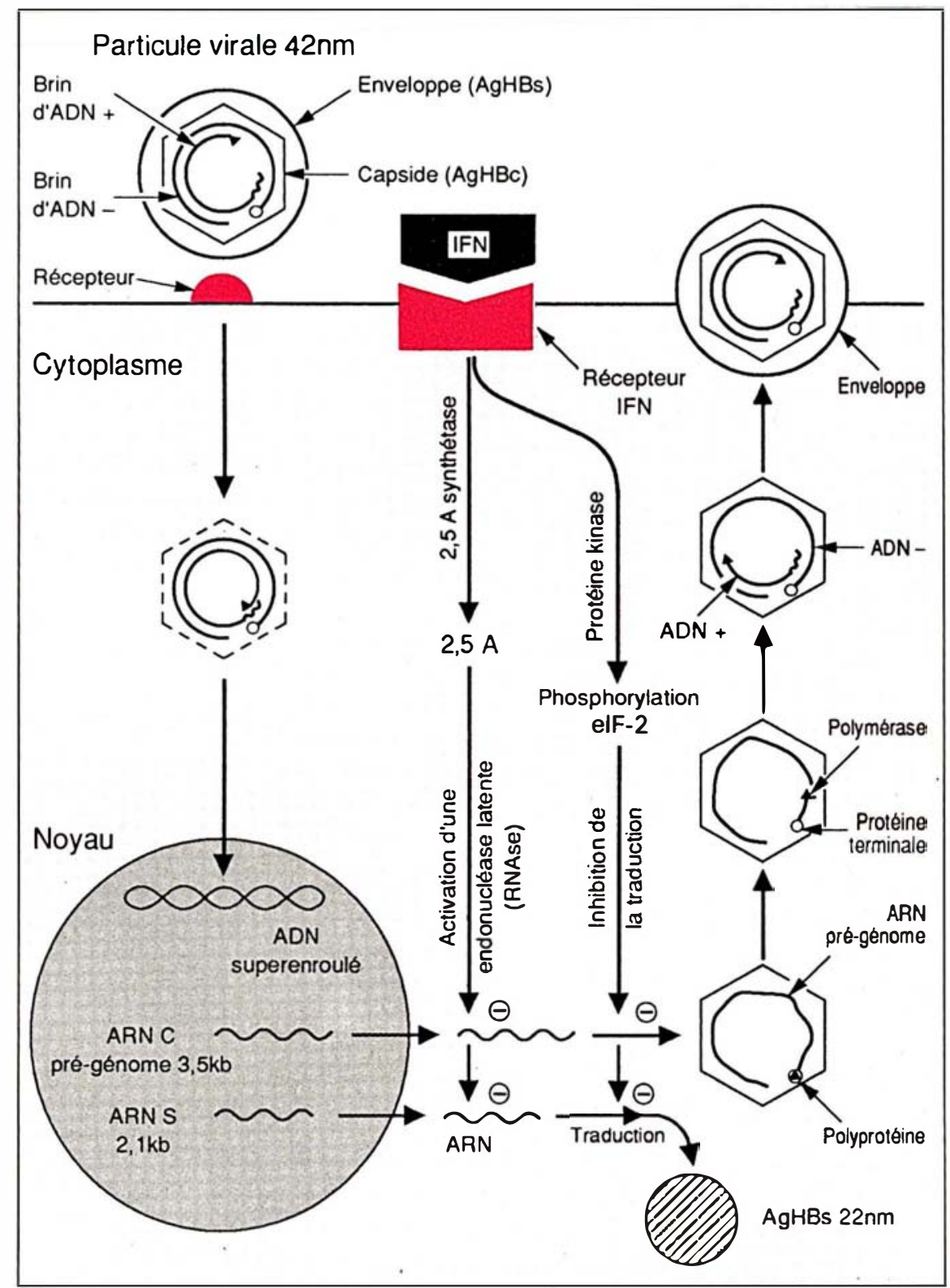

Figure 2. Les sites cellulaires de l'action antivirale de l'interféron. L'interféron se lie au récepteur situé sur la membrane de l'hépatocyte et agit en activant la 2-5-A synthétase. Le 2-5-A synthétisé active une endonucléase nécessaire à la destruction de l'ARNm. L'interféron active aussi une protéine kinase qui inhibe la traduction des protéines virales via la phosphorylation de la sous-unité $\alpha$ du facteur d'initiation de la traduction elF-2.

- L'interféron alpha. Les interférons sont une famille de protéines produites naturellement en réponse à une infection virale. Il en existe trois formes principales : l'interféron $\alpha$, dérivé des monocytes, l'interféron $\beta$, provenant des fibroblastes, et l'interféron $\gamma$, produit par les sous-populations lymphocytaires $\mathrm{T}$ auxiliaires.
Les interférons ont deux types d'action, antivirale et immunomodulatrice [5]. L'action antivirale des interférons (figure 2) passe par leur liaison aux récepteurs cellulaires spécifiques de chaque type d'interféron, induisant toute une série de réponses intracellulaires, dont l'activation de la 2'-5'-oligoadénylate synthétase, et 


\section{RÉFÉRENCES}

11. Fattovich G, Brollo L, Pontisso P, et al. Levamisole therapy in chronic type $B$ hepatitis. Gastroenterology 1986 ; 91 : 692-6.

12. Perillo RP, Regenstein FG, Peters MG, et al. Prednisone withdrawal followed by recombinant alpha interferon in the treatment of chronic type B hepatitis. A randomized, controlled trial. Ann Intern Med 1988 109 : 95-100.

13. Perillo R, Schiff E, Davis GL, et al. Multicenter randomized control trial of recombinant alpha interferon (rIFNa2-b) alone or following prednisone withdrawal in chronic hepatitis B (CHB). Hepatology 1989 . $10: 579$ (abstr).

14. Garcia G, Smith CI, Weissberg JI, et al. Adenine arabinoside monophosphate (vidarabine phosphate) in combination with human leukocyte interferon in the treatment of chronic hepatitis B. Ann Intern Med 1987 107 : 278-85.

15. Perillo RP, Regenstein FG, Bodicky CJ, et al. Comparative efficacy of adenine arabinoside 5' monophosphate and prednisone withdrawal followed by adenine arabinoside 5 ' monophosphate in the treatment of chronic active hepatitis type B. Gastroenterology $1985 ; 88: 780-6$.

16. Caselman WH, Eisenburg J, Hofschneider $\mathrm{PH}$, Koshy R. Beta and gamma interferon in chronic active hepatitis B. A pilot trial of short term combination therapy. Gastroenterology $1989 ; 96: 449-55$.

17. Sherlock S. Treatment of chronic viral hepatitis. J Hepatol 1988; $6: 113-5$.

18. Marcellin P, Ouzan D, Degos F, et al. Randomized controlled trial of adenine arabinoside $5^{\prime}$ monophosphate in chronic active hepatitis B : comparison of the efficacy in heterosexual and homosexual patients. Hepatology 1989 ; 10 : 328-31.

19. Carman WF, Jacyna MR, Hadziyannis S, et al. Mutation preventing formation of hepatitis $\mathrm{B}$ e antigen in patients with chronic l'activation des ribonucléases (qui permettent la destruction de l'ARNm des virions présents dans les cellules infectées). Les interférons possèdent par ailleurs une activité immunomodulatrice (figure 3) : augmentation de l'expression des antigènes de classe I, activation des cellules $T$ impliquées dans la reconnaissance des complexes HLA-épitopes antigéniques viraux et stimulation de l'activité NK. L'interféron semble donc, $a$ priori, être un agent idéal dans le traitement des infections chroniques par le VHB, au cours desquelles existe un défaut de réponse immune au virus et un défaut de production d'interféron.

L'utilisation des interférons lymphoblastoïde et recombinant a, de fait, permis de démontrer l'efficacité de l'interféron $\alpha$, à doses moyennes (3 à $\left.10 \times 10^{6} \mathrm{UI} / 24 \mathrm{~h}\right)$, administré journellement ou mieux tous les deux jours, pendant une durée de trois à six mois, pour obtenir un arrêt de la multiplication du VHB. Dans 30 à $40 \%$ des cas, on observe, à terme, la disparition de l'ADN VHB du sé- rum, l'élimination de l'antigène $\mathrm{HBe}$ circulant, une diminution des transaminases sériques (succédant souvent à une augmentation dans les huit à dix premières semaines du traitement) (figure 4) et une amélioration des lésions histologiques [6]. On peut rarement observer, au décours des traitements par l'interféron, une disparition de l'AgHBs et l'apparition d'anticorps anti-HBs qui indiquerait non pas un arrêt de la multiplication mais une éradication du VHB [7]. Cette guérison complète pourrait survenir préférentiellement chez les patients traités tôt dans l'histoire de l'infection virale $B$, au moment où l'ADN VHB intrahépatocytaire est sous forme non intégrée [8]. Les effets secondaires tels la fatigue, un syndrome grippal, une perte de l'appétit et une fragilisation des cheveux surviennent fréquemment mais sont généralement supportables si les doses administrées ne dépassent pas $5 \times 10^{6} \mathrm{UI} / 24 \mathrm{~h}$.

L'élévation transitoire des transaminases au cours du traitement permet de prévoir l'efficacité de l'interféron. Il semble que la plupart des patients

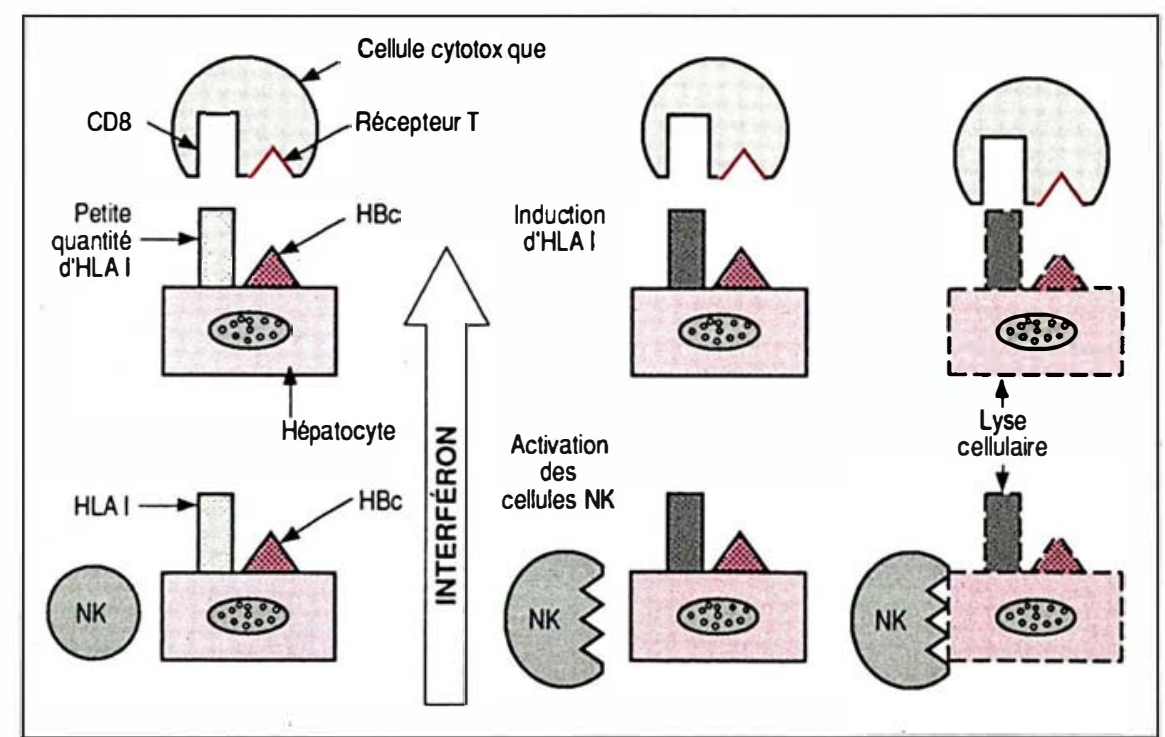

Figure 3. Action immunomodulatrice de l'interféron. Les cellules cytotoxiques détectent les antigènes viraux (HBC) situés à la surface de l'hépatocyte, et présentés par les antigènes de classe I. L'interféron augmente la synthèse des antigènes de classe I, favorisant la reconnaissance par les cellules cytotoxiques. Parallèlement, l'interféron augmente l'activité des cellules NK (natural killer). Ces deux actions favorisent la cytolyse de la cellule infectée par le virus. 
pour lesquels on a observé une guérison complète aient eu une élévation importante des transaminases, supérieure à dix fois la normale au bout de huit à dix semaines de traitement [9].

Cette observation suggère que, pour être efficace, l'interféron doit mettre en ouvre ses deux effets, antiviral et immunomodulateur; l'augmentation des transaminases serait le témoin d'une augmentation de la réponse immunitaire préalable à l'élimination du VHB.

- Autres traitements. L'interleukine-2 et le lévamisole (un immunostimulant) ne sont pas couramment utilisés soit du fait de leurs effets secondaires inacceptables [10], soit de leur relative inefficacité [11].

- Les associations médicamenteuses. Elles ont toutes pour but de combiner les effets antiviraux et immunomodulateurs des différents produits disponibles.

La plus largement étudiée est l'association de corticoïdes à l'interféron [12]. Les corticoïdes administrés aux patients en phase de multiplication virale $B$ ont pour effet d'augmenter encore cette multiplication et de diminuer la réponse immunitaire. L'arrêt de la corticothérapie entraîne un rebond de la réponse immunitaire, qui faciliterait l'action antivirale de l'interféron qui est administré ensuite.

La corticothérapie n'augmente l'efficacité de l'interféron que chez les patients avec faible réponse immunitaire spontanée, indiquée grossièrement par des transaminases peu élevées avant le début du traitement [13].

Les autres associations médicamenteuses n'ont pas montré de supériorité par rapport à l'administration d'interféron seul (interféron-aciclovir, S. Schalm, communication personnelle) ou ont entraîné la potentialisation des effets secondaires sans résultat antiviral intéressant (interféron-vidarabine [14] et corticoïdesvidarabine [15]).

L'association d'interféron $\gamma$ à l'interféron $\beta$ permettrait d'obtenir la suppression de la multiplication virale dans la moitié des cas et la disparition de l'AgHBs comme cela a été observé avec l'interféron $\alpha$ [16].

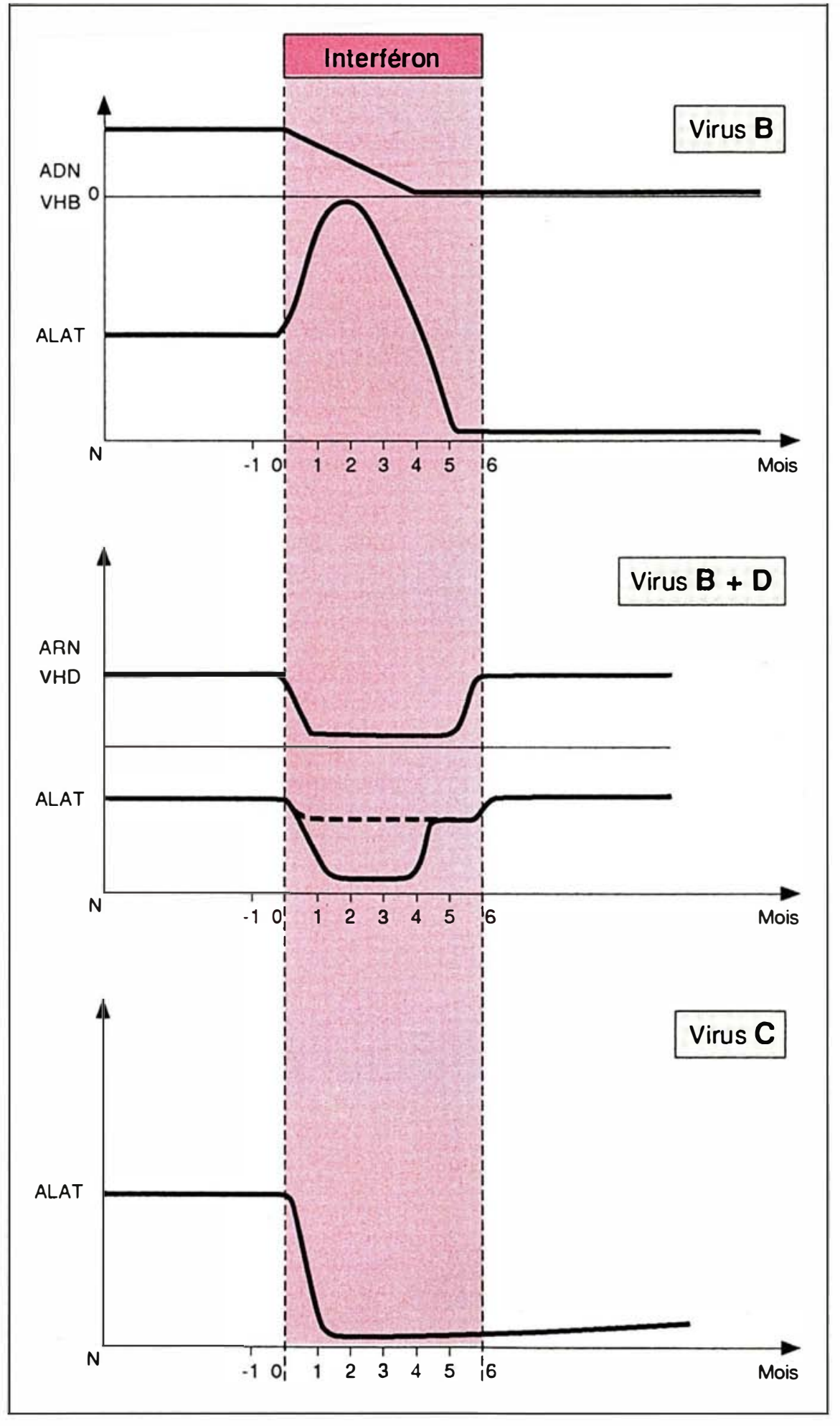

Figure 4. Résultats biochimiques et virologiques de l'administration d'interféron dans les meilleurs cas, au cours des infections par les VHB, $B+D, C . A L A T=$ alanine aminotransférase. A noter que, au cours de l'infection par VHC, la rechute après arrêt du traitement est, en fait, la plus fréquente. 


\section{RÉFÉRENCES}

20. Fattovitch G, Farci P, Brollo L, et al. Anti-HBe and HBV DNA positive chronic hepatitis B : response to interferon. $J$ Hepatol 1989 ; 9 : S29 (abstr).

21. Lai CL, Lok ASF, Lin $\mathrm{HJ}$, et al. Placebo controlled trial of recombinant alpha 2 interferon in chinese HBsAg carrier children. Lancet $1987 ; 2: 877-80$

22. Rizetto M, Verme G. Delta hepatitis present status. J Hepatol 1985 ; 1 : 187-95.

23. Farci $P$, Karayannis $P$, Brook MG, et al. Treatment of chronic hepatitis delta virus (HDV) infection with human lymphoblas toid alpha interferon. Quart J Med $1989 ; 271$ : 1045-54.

24. Rosina F, Sarraco G, Sansalvadore F, et al. Alpha interferon in the treatment of chronic delta hepatitis. Ital J Gastroenterol 1989 ; 21 : 141-5.

25. Choo QL, Kuo G, Weiner AJ, et al. Isolation of a cDNA clone derived from a blood-borne non-A, non-B viral hepatitis genome. Science 1989 ; 244 : 359-62.

26. Alter HJ, Purcell RH, Shih JW, et al. Detection of antibody to hepatitis $C$ virus in prospectively followed transfusion recipients with acute and chronic non-A, non-B hepatitis. N Engl J Med 1989 ; 321 : 1494-500.

27. Esteban JI, Esteban R, Viladomui L, et al. Hepatitis $\mathrm{C}$ virus antibodies among risk groups in Spain. Lancet 1989 ; 2 : 294-7.

28. Van Der Poel CL, Reesink HW, Lelie PN et al. Hepatitis $C$ antibodies and non- $A$, nonB post-transfusion hepatitis in the Netherlands. Lancet $1989 ; 2: 297-9$.

29. Bruix J, Barrera JM, Calvet X, et al. Prevalence of antibodies to hepatitis $C$ virus in spanish patients with hepatocellular carcinoma and hepatic cirrhosis. Lancet 1989 ;
L'examen critique de ces différentes études permet en fait la définition des facteurs prédictifs de la réponse aùx antiviraux et par là de dessiner le profil du patient «bon répondeur » [17].

(a) Le degré de la multiplication virale est manifestement l'élément essentiel, apprécié par la quantification de l'ADN viral circulant, et plus indirectement par le pourcentage de noyaux hépatocytaires exprimant l'antigène $\mathrm{HBc}$ en immunofluorescence; (b) l'importance de la réponse immunitaire (ou l'absence d'immunodépression), qui peut être appréciée grossièrement par l'activité des transaminases ; c'est ainsi que les patients immunodéprimés (infection VIH, insuffisance rénale, transplantation) sont moins bons répondeurs aux traitements antiviraux. Les homosexuels, dans certaines séries, sont moins bons répondeurs [18], peut-être à cause des infections virales à répétition dont ils sont victimes; (c) l'absence d'infection par VIH associée; (d) l'origine ethnique du malade: la réponse aux antiviraux semble moins bonne chez les sujets d'origine asiatique. L'explication de ce fait pourrait être la précocité de la contamination, néonatale (par la mère porteuse chronique du VHB), induisant une tolérance immunitaire (c'est-à-dire une faible réponse) à l'égard du VHB. On rapproche de ce mécanisme le problème des infections néonatales quelle que soit l'origine géographique du patient ; (e) l'état des lésions hépatiques : l'existence d'une cirrhose coïncide généralement avec l'interruption de la multiplication virale, et il n'y a alors pas d'indication à un traitement. Lorsque la multiplication virale n'est pas interrompue chez un patient atteint de cirrhose, un traitement peut être administré en sachant que celui-ci ne fera évidemment pas régresser les lésions histologiques de cirrhose et que l'aggravation transitoire éventuellement entraînée par un traitement antiviral peut avoir de sérieuses conséquences en cas de cirrhose sévère.

Le bon candidat à un traitement antiviral répond donc aux caractéristiques suivantes (Tableau I) : activité forte des transaminases, lésions histologiques d'inflammation portale et nécrose périportale traduisant une activité histologique importante; faible niveau de multiplication virale avant le traitement. Ces signes sont probablement le témoin d'une réponse immune assez forte vis-à-vis du VHB. Les femmes semblent meilleures répondeuses que les hommes, et les individus infectés au cours de la vie adulte sont meilleurs répondeurs que ceux ayant une infection néonatale.

La plupart des études cliniques conduites jusqu'à présent ont porté sur des groupes de patients sélection-

\begin{tabular}{|l|}
\multicolumn{1}{|c|}{ Tableau I } \\
LE CANDIDAT IDÉAL \\
A UN TRAITEMENT ANTIVIRAL \\
\hline $\begin{array}{l}\text { 1. Hépatite B avec antigène HBe } \\
\text { positif }\end{array}$ \\
2. Affection acquise récemment \\
3. Origine européenne (c'est-à-dire \\
ne provenant pas des régions de \\
forte endémie) \\
4. Hétérosexuel \\
5. VIH négatif \\
6. Absence de maladie associée \\
7. Transaminases sériques élevées \\
8. Activité histologique importante \\
dans les régions lobulaires et \\
périportales \\
9. Faible quantité d'ADN VHB séri- \\
que circulant \\
10. Absence d'infection concomi- \\
tante par le virus $\delta$
\end{tabular}

nés sur les critères énoncés plus haut. Il est sûr que les traitements antiviraux méritent d'être évalués dans d'autres groupes de patients, et bien entendu dans le cadre d'études cliniques permettant une exploitation statistique des résultats.

(1) Les sujets négatifs pour l'AgHBe, avec multiplication virale détectée par la présence de l'ADN VHB dans le sérum. On sait que cette situation correspond à un variant du VHB comportant une mutation du gène pré- $C$; cette mutation ne permet pas l'expression de l'AgHBe [19]. Une première étude de l'efficacité du traitement par l'interféron dans ce groupe de patients particulièrement fréquents en Italie a montré une réponse importante à l'administration d'interféron mais aussi un taux de séroconversion spontanée très important dans le groupe témoin ; le bénéfice du traitement, évident après 12 mois, n'est plus statistiquement 
significatif après 24 mois [20].

(2) Les sujets au stade de cirrhose compliquée, qui n’ont pas pu bénéficier des premières études cliniques. Ces sujets ont souvent un faible niveau de multiplication virale. L'efficacité de très faibles doses d'interféron mérite probablement d'être évaluée et il semble qu'une surveillance attentive permette d'éviter les effets secondaires graves du traitement. On tente à l'heure actuelle de traiter les patients atteints de cirrhose avec multiplication virale pour lesquels une transplantation hépatique est envisagée. Il s'agit de réduire le degré de la multiplication virale qui est un facteur pronostique de la récidive de la maladie après la transplantation ; l'évaluation de cette attitude n'est pas encore possible.

(3) Les enfants porteurs chroniques $\mathrm{du}$ VHB ont souvent des lésions hépatiques sévères, et il serait sûrement nécessaire d'entreprendre des études cliniques dans cette population particulière [21].

- Infection virale B-D. L'infection par le virus $\mathrm{D}$ [22] affecte les sujets déjà infectés par le virus $B$, habituellement lorsque la multiplication active du virus B est interrompue. En France et dans les autres pays occidentaux, l'infection par le virus D atteint principalement les toxicomanes. En Italie du Sud et à un moindre degré en Grèce, l'infection par le virus $\mathrm{D}$ est endémique.

L'action antivirale de l'interféron est possible puisqu'on observe une diminution rapide des transaminases sériques chez $55 \%$ des patients traités. Mais une rechute (ré-ascension de la courbe des transaminases) est survenue dans $90 \%$ des cas des malades qui avaient réagi à l'administration d'interféron [23, 24].

\section{Le virus $C$}

- Infection virale C [25-28]. L'infection par le virus $\mathrm{C}$ (VHC) (précédemment désigné sous le terme de virus non $A$, non B) est une cause importante d'hépatite chronique. Il semble bien que le virus $C$ soit directement cytotoxique pour l'hépatocyte. L'histoire naturelle de l'infection par le virus $C$ ne paraît pas comporter les diverses phases décrites dans l'histoire naturelle de l'infection par le virus $B$ : il semble bien qu'il n'existe qu'une seule phase dans cette histoire naturelle, comportant des épisodes intermittents et capricieux de nécrose hépatocellulaire. L'infection virale $\mathrm{C}$ aboutit à la longue, mais dans des délais très différents d'un malade à l'autre, à la constitution d'une cirrhose; lorsqu'une cirrhose est constituée, il existe un risque de carcinome hépatocellulaire [29, 30].

- Traitement de l'hépatite C par l'interféron $\boldsymbol{\alpha}$. La détection des corps anti-VHC vient en fait authentifier les démarches thérapeutiques entreprises depuis plusieurs années dans les hépatites chroniques liées au virus non $\mathrm{A}$, non $\mathrm{B}$. En effet, en se fondant sur l'hypothèse de l'action antivirale de l'interféron $\alpha$, celui-ci a été largement administré à des groupes de patients atteints d'hépatites chroniques non $\mathrm{A}$, non $\mathrm{B}$ posttransfusionnelles et cryptogénétiques. Une première étude avait permis de montrer une diminution rapide de l'activité des transaminases sériques dans un groupe de dix patients [31].

De nombreuses études contrôlées sont venues confirmer ce fait, montrant les faits suivants [32, 33] : (1) la diminution rapide des transaminases chez environ $80 \%$ des patients traités ; (2) la ré-ascension de la courbe des transaminases avant la fin du traitement dans $20 \%$ des cas, et à l'arrêt de l'administration d'interféron dans $50 \%$ des cas traités avec succès, traduisant une rechute de la maladie; (3) l'efficacité de doses moyennes d'interféron $\left(3 \times 10^{6} \mathrm{UI}\right.$ trois fois par semaine), et une certaine relation dose/réponse puisque la réponse obtenue avec des doses inférieures à $3 \times 10^{6}$ UI est moindre. Par ailleurs l'augmentation des doses d'interféron chez les patients mauvais répondeurs au traitement est susceptible d'entraîner une réponse; (4) la durée nécessaire de traitement est d'au moins six mois. La supériorité des traitements prolongés plus de six mois est actuellement en cours d'évaluation ; (5) la comparaison des lésions histologiques avant et après l'administration d'interféron montre une régression des signes de nécrose et d'inflammation appréciés généralement au moyen du score de Knodell [34] ; (6) l'efficacité de l'interfé- ron $\alpha$ est la seule ayant fait l'objet de plusieurs études cliniques. Parmi les différents types d'interféron $\alpha$, recombinant ou lymphoblastoïde purifié, un travail permet d'évoquer la supériorité de l'interféron naturel lymphoblastoïde [35]. L'interféron $\gamma$ n'est pas efficace dans cette indication [36] ; (7) certaines études ont permis de dégager les caractéristiques des patients qui répondent bien au traitement [37, 38] : il s'agit des femmes, d'âge jeune, sans lésions histologiques de cirrhose ; (8) l'étude $a$ posteriori des anticorps anti-VHC dans les séries de patients traités n'a pas permis de montrer une relation entre la réponse au traitement et la présence d'anticorps anti-VHC. De même, il ne semble pas que l'on observe de disparition des anticorps anti-VHC chez les patients traités, ce qui pourrait indiquer la poursuite d'une infection virale. Les titrages des anticorps seraient intéressants pour détecter un éventuel effet immunologique de ce traitement. Toutes ces études sont relativement empiriques puisqu'elles étudient les effets indirects et non virologiques des traitements. La description, encore partielle, du génome du VHC [30] va permettre des études de biologie moléculaire, et les traitements reposeront alors sur des bases plus rationnelles.

Quoi qu'il en soit, il est à l'heure actuelle certain que l'interféron est actif contre le VHC. Les mécanismes virologiques et immunologiques de cette action ne sont pas définis. Le problème majeur pour le médecin est sûrement la fréquence des rechutes à l'arrêt du traitement. Faudra-t-il envisager des traitements très prolongés par des petites doses d'interféron qui semblent bien tolérées, ou associer d'autres traitements?

\section{Conclusion}

Au total l'introduction des médicaments antiviraux a été un grand progrès pour les hépatologues qui jusqu'à présent étaient relativement contemplatifs devant l'aggravation de leurs malades. L'emploi des traitements antiviraux au cours de l'infection par le VHB repose sur des bases maintenant solides, biologiques et virologiques. L'efficacité des traitements est démontrée, mais ne 


\section{RÉFÉRENCES}

30. Colombo M, Kuo G, Choo QL, et al. Prevalence of antibodies to hepatitis $C$ virus in Italian patients with hepatocellular carcinoma. Lancet $1989 ; 2$ : 1006-8.

31. Hoofnagle JH, Mullen KD, Jones DB, et al. Treatment of chronic non-A, non-B hepatitis with recombinant human alpha interfe ron: a preliminary report. $N$ Engl J Med $1986 ; 315: 1575-8$

32. Davis GL, Balart LA, Schiff ER, et al. Treatment of chronic hepatitis $\mathrm{C}$ with recombinant interferon alpha. A multicentric randomized controlled trial. N Engl J Med 1989 ; 321 : 1501-6.

33. Di Bisceglie AM, Martin P, Kassianides C, et al. Recombinant interferon alpha to chronic hepatitis C. N Engl J Med 1989; 321 : 1506-10.

34. Knodell RG, Ishak KG, Black WC, et al. Formulation and application of a numerica scoring system for assessing histological activity in asymptomatic chronic active hepatitis. Hepatology 1981 ; 1 : 431-5.

35. Ideo G, Bellati G, Pedraglio E, Alfieri G. Recombinant alpha 2a interferon (rIFN) lymphoblastoid alpha interferon (L-IFN) or no treatment for non-A, non-B chronic active hepatitis $(\mathrm{CAH})$ : a prospective randomized controlled trial. Hepatology 1989; 10:637 (abstr).

36. Saez-Royuela F, Porres JC, Carreno V. Treatment of chronic non-A, non-B hepatitis with high doses of rIFN-alpha of rIFN gamma. Hepatology 1989 ; $10: 646$ (abstr).

37. Di Bisceglie AM, Lisker-Melman M, Martin $\mathrm{P}$, et al. Factors predicting the outcome of alpha interferon therapy for chronic non-A non-B hepatitis. Gastroenterology 1989; 86 : A593 (abstr).

38. Causse X, Godinot H, Ouzan D, et al. Recombinant alpha interferon for chronic non-A, non-B hepatitis/optimal doses and factors associated with favorable response.

Hepatology 1989; $10: 643$ (abstr). porte pas sur l'ensemble des malades puisque les succès sont observés au mieux dans $40 \%$ des cas. Lorsqu'un bon résultat est obtenu, la disparition de la multiplication virale et des signes histologiques d'activité de la maladie est durable et permet la prévention de la survenue d'une cirrhose et de ses complications. Il existe même des arguments virologiques pour proposer un traitement précoce de l'infection virale chronique (après six mois de présence des marqueurs sériques du VHB), avant l'intégration du virus au génome de l'hôte. Il est donc clair qu'un traitement antiviral doit être proposéà tout patient répondant aux conditions de «bon répondeur » définies plus haut. Mais ces traitements restent lourds, parfois complexes (associations médicamenteuses), les effets secondaires doivent être surveillés, et il est donc plus prudent de mettre en route de tels traitements en milieu spécialisé. Par ailleurs, les modalités thérapeutiques concernant les groupes de moins bons répondeurs (enfants, sujets atteints de cirrhose) et les malades atteints d'hépatites à variants du VHB, ainsi que les infections par les virus $B$ et $\delta$, doivent encore faire l'objet d'études cliniques.

En cas d'hépatite liée à un virus non $A$, non B, il semble que les arguments épidémiologiques, cliniques et histologiques soient actuellement suffisants pour proposer un traitement, sans tenir compte de la présence des anticorps anti-VHC, puisqu'on n'a pas trouvé de relation entre la présence d'anticorps antiVHC et la réponse à l'interféron. Il n'y a pas non plus de différence selon le mode de contamination (posttransfusionnel, toxicomanes ou « cryptogénétique ») et un traitement peut être proposé dans tous les cas. Le vrai problème reste l'instabilité de la réponse, qu'elle soit jugée sur le plan biochimique (transaminases) ou histologique. Des protocoles thérapeutiques sont actuellement en cours en Europe et aux États-Unis (non A, non B dans le cadre de protocoles thérapeutiques, et non en traitement « ouvert»).

C'est au prix de cette discipline de la part des médecins et des malades que les conditions et les modalités optimales de tels traitements pourront être rapidement précisées

\section{Summary}

Treatment of chronic hepatitis

Treatment of chronic hepatitis due to infections by virus $B$ and $\mathrm{C}$ are rewieved. In chronic HBV infection, the treatment can be used only in patients with active HBV replication, i.e. with presence of $\mathrm{HBeAg}$ and/or HBV DNA. Vidarabin, a nucleoside analogue, is efficient in about $30 \%$ of cases but has severe sideeffects. Alpha interferon can be used alone or associated with steroids. The response rate is $40 \%$, with inhibition of viral replication and, in some cases, seroconversion from $\mathrm{HBsAg}$ to anti-HBs. Patients are more likely to respond if transaminases are high, inflammation and necrosis are present on the liver biopsy and the level of viral replication is low. Antibodies to the recently described HCV can now be detected in the serum. Low doses of interferon are efficient in $80 \%$ of the patients with chronic hepatitis due to HCV and elevated transaminases, but relapses with elevation of transaminases occur in most of the cases when the treatment is discontinued.

\section{TIRÉS A PART}

F. Degos. 